\title{
Intelligent Fluorescence Image Analysis of Giant Unilamellar Vesicles and Protein Liquid Phase Droplets by Whole Z-stack Analysis
}

II-Hyung Lee ( $\sim$ leei@montclair.edu )

Montclair State University

Sam Passaro

Montclair State University

Selin Ozturk

Montclair State University

Weitian Wang

Montclair State University

\section{Research Article}

Keywords: Phase separation, Lipid membrane, Lipid raft, Membrane organization, Protein phase, Fluorescence imaging, Membrane reconstitution, Deep learning

Posted Date: March 1st, 2021

DOI: https://doi.org/10.21203/rs.3.rs-242107/v1

License: (9) This work is licensed under a Creative Commons Attribution 4.0 International License. Read Full License 


\section{Abstract}

Fluorescence image analysis in biochemical science often involves the complex tasks of identifying samples for analysis and calculating the desired information from the intensity traces. Analyzing giant unilamellar vesicles is one of these tasks. Researchers need to identify many vesicles to statistically analyze the degree of molecular interaction or state of molecular organization on the membranes. This analysis is complicated, requiring a careful manual examination by researchers, so automating the analysis can significantly aid in improving its efficiency and reliability. We developed an intelligent analysis routine based on the 3D information of whole z-stack images. The program identifies the valid vesicles to analyze and calculates the desired data automatically. The program can examine the amount of protein binding on the membranes and determine the state of domain phase separation by calculating the fluorescence intensity trace along the membranes. We also show that the method can easily be applied to similar analyses, such as intensity analysis of phase-separated protein droplets. A deep learning-based classification approach enables the identification of vesicles even from relatively complex samples. We demonstrate that the proposed artificial intelligence-assisted classification can further enhance the accuracy of the analysis close to the performance of manual examination.

\section{Introduction}

A giant unilamellar vesicle (GUV) is an artificial lipid membrane system characterized by its micrometerwide globular shape. Because GUVs have the advantage of deformability and a relatively large size, they have been used in a variety of biophysical and biochemical experiments. ${ }^{1}$ Example studies include the study of lipid membrane phase separation, ${ }^{2-4}$ phase modulation by membrane anchored proteins, ${ }^{5,6}$ reconstitution of membrane remodeling processes, ${ }^{7,8}$ the viral assembly process, ${ }^{9}$ measurement of mechanical tension, ${ }^{10,11}$ effect of osmotic pressure, ${ }^{12}$ membrane protein reconstitution, ${ }^{13}$ and monitoring protein binding to the membrane. ${ }^{14}$

Fluorescence imaging and fluorescence image analysis are extensively used in these types of experiments. The most common mode of analysis is manually studying each individual vesicle to examine enough vesicles for statistical analysis from a population of GUVs. ${ }^{5}$ Although this may be suitable for experiments with a relatively small number of GUVs, for larger-scale sample numbers, manual analysis is very time consuming and often difficult to implement, particularly when researchers are not very experienced with the nature of the GUV system and fluorescence imaging. Automated analysis can provide an effective solution to these problems by standardizing the analysis procedure. GUV image analysis is complicated enough that it requires well-designed algorithms to automate the analysis to the comparable level that can be performed by a trained researcher, and there has been some effort to address similar problems by other groups. ${ }^{15-18}$

In 2014, Hermann et al. introduced the circular Hough transformation (CHT) algorithm for the automated detection of GUVs in fluorescence images. ${ }^{15} \mathrm{CHT}$ is an algorithm originally developed to detect circular 
objects from complex images in computer science. ${ }^{19}$ Briefly, $\mathrm{CHT}$ first converts the original image into an edge image to clearly define the edge of objects. Basing on the edge image, it starts the voting process to score the likelihood of having a circle with radius $r$ at any given pixel position. The pixels at the center of circular objects appear as high-score pixels in the transformed image space; thus, the position of circles with radius $r$ can be decided based on likelihood. If radius $r$ is to be flexibly decided, radius $r$ also becomes a parameter of the likelihood voting process, which makes it computationally more expensive. Fluorescence images of GUVs usually show very clear boundaries of GUV contours, so CHT is an excellent method to automatically detect individual GUVs or any other similar circular objects from the fluorescence image. CHT has been widely used and improved, so it is also readily available to apply in commonly used mathematical software and programing languages.

In this report, we extended the $\mathrm{CHT}$ detection algorithm by developing a deep learning-based approach for the selection and intensity analysis of full GUV image stacks, which are a collection of image sections along the z-axis, including the information of full 3D GUVs. An intensity analysis algorithm was developed to study protein binding and phase separation state decisions. We applied automated analysis methods to examine several typical sets of data. In the binding analysis, we show how his-tagged protein bound to Ni-DGS lipid can be detached by competitive binding of imidazole solution. In the phase state analysis, we present how the existence of a separated lipid phase can be detected by contour intensity analysis. In addition to the GUV analysis, we applied the $\mathrm{CHT}$ detection algorithm to automatically analyze fluorescence images of liquid phase-separated protein droplets. Proteins with multivalent binding interaction or intrinsically disordered domain interaction at a high concentration spontaneously form protein droplet domains that may serve as independent organelles in our cells. ${ }^{20-30}$ Given the appreciation of thermodynamic phase separation as a general principle of protein sorting in the field, in vitro reconstitution experiments of protein droplets have become very common these days. Protein droplets have sizes and shapes comparable to those of GUVs, so a similar automated analysis may be introduced for the high-throughput analysis of protein droplet domains. We show how the $\mathrm{CHT}$ algorithm could be successfully applied to protein domain analysis, and we also present an example analysis of differences in protein cargo concentration at different conditions.

Lastly, we show how a deep learning approach can be leveraged for the problem as a complementary and potentially more capable solution. Deep learning is a process in which an artificial network is trained with sample images, so it can recognize different images without specifically designing and extracting features. It has been proven to be powerful in descriptor extraction and classification for visual recognition tasks. ${ }^{31}$ Because of recent progress in deep learning, its capability and efficiency reach even beyond human recognition, and it has been applied to a wide range of related fields in leading-edge science and engineering. ${ }^{32-40}$ As our work involves the classification of images, we integrated this approach into our algorithm for enhanced performance. We show an efficient implementation of deep learning in vesicle classification for choosing the right vesicles.

\section{Method}




\section{CHT-based GUV stack analysis}

The first step of the analysis is to identify the GUVs and apply selection filters in order to choose GUVs with the right condition. In fluorescence imaging, the images of 3D objects are collected as z-stacks or a series of image sections at different heights. In some simple analyses, examining single section images may be sufficient, but whole stack analysis ensures that we are studying the entire 3D information of GUVs, and some filtering strategy and analysis are only possible when we have access to the whole 3D information.

Z-stacks are analyzed from the first to the last images one by one, as shown in Figure $1 \mathrm{a}$. In each image, the $\mathrm{CHT}$ algorithm is applied to detect GUV fluorescence circles. The sensitivity parameter of CHT detection can be adjusted to change the strictness of circle definition, and the radius range can also be set to only detect GUVs with a reasonable size. Once all GUV fluorescence circles are detected from each section of the image, 3D GUVs are identified by grouping fluorescence circles by their center positions. All fluorescence circles with center $(x, y)$ positions within the set range are considered as one GUV and are grouped as an individual GUV entity. Each grouped GUV is recorded as a matrix containing the position, radius, and intensity of each image section that can be used for further analysis.

GUV selection filtering strategies are applied in two separate stages, as shown in Figure 1b. The first filtering is applied after detecting GUV fluorescence circles from each image $\mathrm{CHT}$. In this stage, the contrast between the circular edge and the internal space is used to filter out ill-defined circles. The intensity per pixel is calculated for pixels near edges and pixels inside the circle, and any circles with insufficient relative intensity near edges are filtered out. This way, the images of multi-lamellar vesicles or vesicles with too much fluorescence content can be filtered out. GUV circles that are not clearly analyzable using the intensity contour because of ambiguous positions can be removed in this stage, as well. The second filtering strategy is done after grouping individual GUVs. In this stage, any GUVs with insufficient information are filtered out. Specifically, GUV groups with only one or two image sections are filtered out because they do not have enough images to be considered as an intact GUV.

The result of the initial $\mathrm{CHT}$ detection and filtering is displayed and recorded visually, so users can adjust the selection parameters to use until the outcome shows the most reliable detection result for the purpose of analysis.

\section{Intensity binding analysis}

The purpose of intensity binding analysis is to quantify the change in fluorescence intensity on the membrane in a fluorescence channel for any membrane binding species. Reference channel $A$, in which the fluorescence signal comes from the lipid membrane, is used to detect and group GUVs to analyze; analysis channel $\mathrm{B}$, in which the fluorescence signal comes from interacting species, such as membrane binding proteins, is analyzed for changes in the fluorescence intensity signal on the membrane. 
For all fluorescence images that passed selection strategies, the matching fluorescence intensity of channel $B$ is analyzed using the position and radius information obtained from channel $A$. The fluorescence intensity of all pixels adjacent to the circle defined by the center position and radius is summed up, as shown in Figure $2 \mathrm{a}$. The total intensity values and intensity per pixel values are recorded for all images. The $\mathrm{CHT}$ algorithm used in this study detects each circle in such a way that the radius is slightly greater than the actual intensity maxima of the fluorescence circle, so counting pixels that are $n$ pixels, by user definition, inward successfully sums up all intensity values. As each GUV is grouped, the intensity per pixel from each GUV can be calculated.

To account for the background signal, the net intensity is defined as follows in Eq. 1:

$$
I_{\text {net }}=I_{\text {raw }}-I_{\text {background }}
$$

This means that the general background signal per pixel was estimated and subtracted from the raw intensity signal. The background signal can be estimated by measuring the average signal coming from the dark part of the images. To make this process more systematic, we introduced automated methods to the program. In the automated estimation, a background signal is estimated based on the analysis of the intensity histogram of each image. Users may choose to use the most common intensity value or the triangular thresholding estimation value. ${ }^{41}$ Both worked fine for most analyses we tried, but triangular thresholding is recommended, which uses the most probable intensity values from both background and net fluorescence signals.

\section{Binary masking for high-sensitivity analysis of low signal-to-noise ratio images}

Background intensity estimation was not only used to correctly calculate the net intensity signal but also to create a binary mask of the image to analyze. We learned that creating a binary mask, which is an image version in which the original image is converted into 1 or 0 image based on the estimated threshold intensity, significantly enhanced the performance of circular detection in $\mathrm{CHT}$, especially when the signal-to-noise ratio of the image is relatively low. To make the $\mathrm{CHT}$ method generally applicable to images with any signal-to-noise ratio, binary masking was used for all the methods described. Successful examples of circular detection using binary masking are shown in Figure $2 b, c$.

\section{Phase separation determination using intensity contour analysis}

The purpose of phase separation determination analysis is to systematically identify the binary phase state of GUVs, homogeneous or phase separated, using a well-defined definition for the statistical analysis of a population of GUVs. In addition to the phase determination, the analysis also calculates and reports the contour intensity of each z-section image of vesicles and the fluorescence intensity of different phases that may be used to calculate the partition coefficient of the reporter molecule. 
The analysis first detects individual GUVs using the general CHT circular detection and filtering strategies to find qualifying GUVs to analyze. Example GUV detection results are shown in Figure $3 a, b$ for a typical uniform GUV sample with most of GUVs in a uniform phase state and a phase-separated GUV sample with most of GUVs in a phase-separated state. Binary masking CHT detection followed by the GUV stack analysis successfully detected GUVs to analyze in typical phase separation samples. Intensity contour analysis uses the intensity around the circular perimeter of each section image to make a decision about phase separation. If the GUV is phase separated, the intensity around the perimeter will show discrete discontinuity at the phase boundary, as shown in Figure 3c. If the GUV is uniform without phase separation, the intensity plot will not have any noticeable discontinuity around the perimeter, as shown in Figure $3 \mathrm{~d}$. As this decision is made for each section of a GUV, the decision parameter can be set to make a final state decision for each GUV. For example, a GUV may be determined as a phase-separated vesicle when at least $60 \%$ of the z-section images show clear phase separation.

For contour intensity analysis, the intensity around the perimeter was divided into $\mathrm{N}$ segments. The average net intensity per pixel was calculated for each segment by using the pixels within $(r-\Delta r, r)$ and $(\theta$, $\theta+360 / N$ ), where $r$ and $\theta$ indicate the radius and the angle in a polar coordinate of a circle image, respectively. For each contour intensity segment, histogram analysis is performed by aligning segments by average intensity from the highest intensity to the lowest one. The segment intensity of the $20 \%$ from the highest is defined as high intensity, and the segment intensity of the $20 \%$ from the lowest or $80 \%$ from the highest is defined as low intensity. These are estimated intensities of the fluorescence intensity of two different phase domains. The mid-intensity between the high intensity and the low one is calculated, and discontinuity analysis is performed along the direction of the increasing degree $\theta$ to determine whether there is any directional decrease or increase in intensity spanning $\pm p \%$ from the mid-intensity (Figure 3c, d). The $p$ value may be adjusted based on the partition coefficient of the fluorescent report used. For a high partition coefficient reporter, which strongly prefers one domain over the other, a higher $\mathrm{p}$ may be used. For a low partition coefficient reporter with a relatively even distribution but with a clear preference for one domain over the other, a smaller $p$ value should be used. High and low intensity estimation from the $20 \%$ percentile may be adjusted if necessary, but choosing a too small percentile is not recommended, as it may select a false high or low intensity coming from an outlier intensity. Choosing an overly large percentile may fail to correctly select high and low intensity in the histogram distribution when the relative proportion of one domain is small. Through an analysis of the number of discontinuities in the intensity contour, the number of domains may be estimated if needed, when finely modulated phase behavior with many domains per vesicle is expected, as opposed to a binary segregation with only two domains.

\section{Protein droplet intensity analysis}

Protein droplet phase separation is another phase separation behavior that is commonly studied with an in vitro reconstitution experiment. Because of the comparable size and globular shape of the protein droplets to the GUVs, the same analysis method can be applied to automatically calculate the fluorescence intensity of each droplet. CHT analysis can detect circular images from z-section images, and it can be reconstituted to find individual droplets. The fluorescence intensity of each droplet is 
analyzed by calculating the net average intensity per pixel of all the pixels within the circles that can be used to quantify the amount of protein in the droplet. ${ }^{21}$ Background intensity is subtracted to correctly calculate the net intensity of the droplets, as specified in Eq. 1. Some droplets that are at the stage of two droplets coalescing with each other may be detected as two droplets that are very close to each other. Such cases can be avoided by ruling out all overlapping droplets, or they may just be analyzed as individual droplets.

\section{Deep learning-based image filter}

The purpose of a deep learning-based image filter is to enhance the capability of the GUV image selection strategy so that the program can recognize even more complicated cases of images that a simple calculation algorithm might miss. The convolutional neural network (CNN), a powerful and reliable network of deep learning, was used in this study to achieve this objective. Using the training images and defined classes, the $\mathrm{CNN}$ is trained by minimizing the mean squared error between the inputs and outputs. The $\mathrm{CNN}$ is mainly structured with the normalization layer, the convolutional layer, the rectified linear unit (ReLu) layer, the pooling layer, and the fully connected layer. During the training process, the sample images will be inputted to the normalization layer, which performs image normalization. The convolutional layer is applied for feature extraction. The ReLu layer will introduce nonlinearity to the CNN model. The pooling layer will reduce the image spatial dimension. The fully connected layer is used for high-level reasoning in the neural network. A total of 5,853 training images of individual vesicles were used. The images were converted into $50 \times 50$ pixels as inputs for the CNN. Each image represented a possible z-section image of a GUV. The images were classified into four classes. In the training process, these four classes were defined as C1-valid vesicles, C2-multi-lamellar vesicles, C3-vesicles overlapped with other vesicles, and C4-hazy or unclear images. A total of 1,918 training images of $\mathrm{C} 1,877$ of $\mathrm{C} 2$, 1,129 of $\mathrm{C} 3$, and 1,929 of $\mathrm{C} 4$ were used. The neural network was trained and validated using the training set. Then, the trained CNN model takes unlabeled images as the input and generates the corresponding classes. In this new version of the phase determination program, instead of the vesicles being filtered based on intensity calculation, each detected vesicle image was converted into $50 \times 50$ images for classification by the trained neural network. Only images of $\mathrm{C} 1$-valid vesicles passed the enhanced filtering strategy. The rest of the calculation was identical to the previously explained algorithm for vesicle state determination.

\section{Sample preparation}

GUV was prepared with the electroformation method. ${ }^{42,43}$ Briefly, a lipid mixture of a desired composition was deposited on an indium tin oxide (ITO)-coated glass (Delta Technologies) at $55^{\circ} \mathrm{C}$. The lipid was dried by nitrogen gas and further dried in a vacuum chamber. The glass, along with another ITO glass, formed a sealed chamber separated by a silicon spacer (Grace Bio-Labs), and sucrose solution was introduced. Sinusoidal voltage of $2 \mathrm{~V} / \mathrm{mm}$ space at $5 \mathrm{~Hz}$ was applied for about $2 \mathrm{~h}$ at $55^{\circ} \mathrm{C}$ to induce GUVs. The GUVs were collected and used in one day. The lipids used in this study were from Avanti Polar Lipids. The proteins utilized in this research were purified by E. coli overexpression, followed by Ni affinity 
purification and gel filtration. The plasmids were given by Michael Rosen (Addgene plasmid \# 127093, \#126946, and \#127093). ${ }^{21}$ The imaging samples were prepared similar to the method described in ${ }^{5}$. Briefly, an AttoFluor cell chamber (Invitrogen) and a cover glass cleaned by bath sonication in isopropyl alcohol: water $=1: 1$ were assembled. The glass surface was blocked by incubating with $5 \mathrm{mg} / \mathrm{mL}$ BSA solution for $30 \mathrm{~min}$. After incubation, the chamber was washed five times with the buffer for the addition of the GUV solution for imaging. To analyze protein interaction, the desired concentrations of proteins were added by pipette injection and mixed gently. To ensure a quick and homogeneous interaction, the protein solution was added at a volume comparable to that of the solution in the chamber $(20 \%-50 \%$ by volume). Protein droplet samples were first mixed in a test tube to incubate for at least $30 \mathrm{~min}$, and they were introduced to the chamber for imaging. To decrease the volume of the chamber, a silicon 0-ring was introduced.

\section{Imaging condition}

All the images shown in this report were collected using confocal fluorescence laser scanning microscopy unless specified otherwise. Briefly, a Nikon Ti-E-based C2 confocal microscope was used. Excitation laser lights of 488 and $561 \mathrm{~nm}$ were used with matching emission filters to collect signals from the fluorescent molecules. A Nikon Plan Apo 100× NA 1.45 oil immersion objective was used without further magnifying the lens in the optical path. The typical mode of scanning was to collect data as 1,024 $\times 1,024$ pixels spanning a $127.3 \mu \mathrm{m} \times 127.3 \mu \mathrm{m}$ area, whereas motorized $\mathrm{z}$-axis movement allowed the automated acquisition of z-stack images.

\section{Software platform used}

GNU Octave, a freely available scientific programming language, was used to test all the non-deep learning algorithms shown in this report. The algorithm and the strategy we tested in this report are generally applicable in any language, but we chose this platform because it is freely available to researchers, the language is intuitive and approachable for students in training to learn, and exact procedures of the subroutines used can be easily examined. The version we used in this project was completed with GNU Octave 5.1.0 and its image package. A deep learning-based algorithm was developed and implemented in MATLAB R2020b (MathWorks) with image processing and deep learning toolboxes. All raw codes in this article are available as supplementary materials with example images.

\section{Results}

\section{Detecting the unbinding of his-tagged fluorescent proteins from the membrane by imidazole inhibition}

We tested multi-channel intensity-based binding analysis by examining data from relatively simple binding and unbinding experiments of fluorescent proteins on the GUV membrane. In this experiment, histagged green fluorescence protein (GFP) was bound to the GUV with functionalized Ni-DGS lipids by incubating the sample with the protein. The protein was then detached by introducing a high concentration $(300 \mathrm{mM})$ of imidazole, without altering the osmolality of the buffer, to detach the proteins 
from the membrane via inhibition of $\mathrm{Ni}$-his tag interaction. We analyzed GFP fluorescence intensity on the membrane before and after introducing the inhibitor. $\mathrm{CHT}$ detection found circular fluorescence using the TR-DHPE fluorescent lipid signal, and qualifying GUVs after filtering strategies were used to quantify the fluorescence signal from the GFP protein on the membrane in the protein fluorescence channel. Multiple GUVs under the same conditions were quantified, and the average value was calculated. As shown in Figure 4a, the intensity-based binding analysis successfully quantified the difference before and after introducing the imidazole inhibitor to the sample. The amount of his-tagged GFP protein on the membrane after introducing the inhibitor was less than $5 \%$ of the intensity before introducing the inhibitor, suggesting that the majority of proteins that were originally bound to the membranes were removed from the membrane as a result of inhibition.

\section{Statistical analysis of phase state}

We tested the contour intensity-based phase state determination program by analyzing samples with relatively clear differences in phase behavior. GUVs with uniform phase were prepared as a 1,2-dioleoylsn-glycero-3-phosphocholine (DOPC): 1,2-dioleoyl-sn-glycero-3-[(N-(5-amino-1carboxypentyl)iminodiacetic acid)succinyl] (Ni-DGS) $=90: 10$ mol composition; $0.2 \mathrm{~mol} \%$ of DOPC was replaced by TopFluor-labeled cholesterol (TF-Chol), a fluorescence reporter. GUVs with a highly phaseseparated state were prepared as a DOPC:Ni-DGS: 1,2-dipalmitoyl-sn-glycero-3-phosphocholine (DPPC): Cholesterol = 15:10:50:25 mol composition; $0.2 \mathrm{~mol} \%$ of chol was replaced by TF-Chol, a common fluorescence reporter of the same mol composition in both sets of GUVs. With an appropriate adjustment of parameters to use in the automated analysis, the program successfully detected and statistically distinguished two samples, as shown in Figures $4 \mathrm{~b}$ and c. Individual decisions were visualized by saving color-coded images during the analysis, and we could review the saved images to validate the reliability of the state decision for each individual vesicle. Most of the false decisions could be avoided by abandoning ambiguous GUVs; however, occasional false decisions included judging GUVs that are in contact with another GUV as a vesicle with phase-separated domains, with the use of false contour intensity coming from a double-layered GUV with two bilayers very closely spaced to each other. Furthermore, contour analysis could not analyze the information of the image at the very top and at the bottom, as it shows phase state information as phase behavior happening on the plane instead of at the perimeter. This may be valuable information for human researchers, but this version of contour analysis will not be able to use this information. Nevertheless, the performance of the program was generally reliable and unbiased.

\section{Protein droplet intensity analysis at different cargo concentrations}

We tested the protein droplet intensity analysis program by analyzing protein droplet samples prepared at different concentrations of fluorescent cargo proteins. As shown in Figure $5 \mathrm{a}$, the same approach of the automated analysis of CHT-based circle detection, followed by the whole stack analysis, selected individual protein phase droplets very effectively. The example analysis was performed on protein droplets formed between two multivalent binding partners, SUMOx10 repeats and SIMx10 repeats, at 
about $10 \mu \mathrm{M}$ concentrations each. GFP-SUMO3, a fluorescent protein with three repeated SUMO domains, was introduced as a cargo protein that reported the protein droplet clearly. The cargo protein concentration was varied at a relatively low concentration range from 10 to $100 \mathrm{nM}$. At all concentrations, droplet detection was efficient, and fluorescence intensity showed a monotonic increase from 20 to 100 $\mathrm{nM}$ concentration regions, although it did not show a noticeable difference in intensity from 10 to $20 \mathrm{nM}$. (Figure 5b)

\section{Deep learning-based GUV selection strategy for enhanced vesicle identification}

The deep learning-based approach uses CNN trained by the sample images. The sample images consist of pre-classified classes of images as the input. The CNN is trained until it can successfully classify images that were not part of the training sample images. This approach uses artificial intuition by pattern recognition of the objects, so it is potentially more capable when the objects to classify are difficult to define with a simple calculation-based strategy. Figure 6a shows the typical images in each class of images we used to train the neural network. Class 1 represents the valid vesicles to analyze; other classes represent vesicles that should be excluded for various reasons. Class 2 represents mutilamellar vesicles or vesicles that contain unexpected lipid membrane structures inside; class 3 represents vesicles that are too crowded, which makes the analysis of an individual vesicle ambiguous; and class 4 represents vesicles with a too low image quality to analyze mostly because the contour of the lipid membrane is not fully in focus.

When the trained network was used to detect vesicles images, the filtering strategy very strictly excluded vesicles that had any features represented by classes $2-4$, as shown in Figure $6 \mathrm{~b}-\mathrm{c}$. Some vesicle images had multiple features of classes $2-4$, such as a multi-lamellar vesicle with a hazy intensity trace.

Therefore, some vesicles could not be classified uniquely, but the vesicles to be filtered almost always fell under one of classes 2-4, allowing only valid vesicle images of class 1 to survive the selection strategy. Combined with the whole z-stack approach, the likelihood of invalid vesicles surviving the automated selection strategy was very small, and valid GUVs were successfully selected. This approach was especially beneficial to exclude some cases that were ill defined by simple calculation. Multi-lamellar vesicles that have multilayers only near the outermost vesicle membranes and crowded vesicles in which a part of the membrane is touching other lipid membranes are examples.

\section{Discussion}

We conducted an intelligent fluorescence image analysis based on whole z-stack images of GUVs. Our approach is unique in that it can effectively select the valid vesicles to analyze from a population of vesicles and perform fluorescence intensity-based calculation on various pieces of information, such as the amount of lipid-protein interaction and the phase state of vesicles. The proposed automated selection and standardized calculation can also facilitate numerous large-scale and complex image analyses and statistics in both science and engineering areas. Our method can detect vesicles from relatively low signal-to-noise ratio samples, and it can easily be expanded to various applications of 
multi-channel intensity analysis. We also showed that the method of automated detection can be used for protein droplet analysis, which we believe will be especially useful when studying the interaction between lipid vesicles and protein phase droplets. Deep learning-based classification could successfully recognize vesicle types for the purpose of excluding unwanted vesicles. To the best of our knowledge, this is the first implementation of deep learning to the problem. Deep learning is potentially a very powerful approach because it can classify very complicated classes as long as an artificial neural network is well trained. However, its performance strongly depends on the quality and amount of training image sets, so a reasonable definition of classes with image samples spanning many variations of each class will be important for the training and validation accuracy. As typical image analysis in the field requires a process in which researchers identify each individual entity and make a decision on the class of each, the application of the deep learning approach can greatly improve the efficiency and reliability of the analysis. A deep learning-based decision is also fast, and in the long term, it can output class decision information in real time as the data are being collected, providing a dynamic feedback platform to enhance the effectiveness and quality of the experiment.

\section{Declarations}

\section{Acknowledgements}

The authors would like to thank Dr. Laying Wu of the Microscopy and Microanalysis Research Laboratory at Montclair State University for the microscope training and facility maintenance. The authors are also grateful to Miranda Roland (University of Puget Sound) for contributing some preliminary data that helped this research. The study was supported by the startup fund and the Sokol student scholar research fund of the College of Science and Mathematics, Montclair State University.

\section{References}

1 Chan, Y.-H. M. \& Boxer, S. G. Model membrane systems and their applications. Current Opinion in Chemical Biology11, 581-587 (2007).

2 Veatch, S. L. \& Keller, S. L. Separation of Liquid Phases in Giant Vesicles of Ternary Mixtures of Phospholipids and Cholesterol. Biophys J85, 3074-3083 (2003).

3 Goh, Shih L., Amazon, Jonathan J. \& Feigenson, Gerald W. Toward a Better Raft Model: Modulated Phases in the Four-Component Bilayer, DSPC/DOPC/POPC/CHOL. Biophys J104, 853-862 (2013).

4 Balleza, D. et al. Complex Phase Behavior of GUVs Containing Different Sphingomyelins. Biophys J116, 503-517, doi:10.1016/j.bpj.2018.12.018 (2019).

5 Lee, I.-H., Imanaka, M. Y., Modahl, E. H. \& Torres-Ocampo, A. P. Lipid Raft Phase Modulation by Membrane-Anchored Proteins with Inherent Phase Separation Properties. ACS Omega4, 6551-6559, 
doi:10.1021/acsomega.9b00327 (2019).

6 Scheve, C. S., Gonzales, P. A., Momin, N. \& Stachowiak, J. C. Steric Pressure between MembraneBound Proteins Opposes Lipid Phase Separation. Journal of the American Chemical Society135, 11851188, doi:10.1021/ja3099867 (2013).

7 Roux, A. et al. A minimal system allowing tubulation with molecular motors pulling on giant liposomes. Proceedings of the National Academy of Sciences99, 5394, doi:10.1073/pnas.082107299 (2002).

8 Schöneberg, J. et al. ATP-dependent force generation and membrane scission by ESCRT-III and Vps4. Science362, 1423, doi:10.1126/science.aat1839 (2018).

9 Carlson, L.-A., Bai, Y., Keane, S. C., Doudna, J. A. \& Hurley, J. H. Reconstitution of selective HIV-1 RNA packaging in vitro by membrane-bound Gag assemblies. eLife5, e14663, doi:10.7554/eLife.14663 (2016).

10 Tian, A., Johnson, C., Wang, W. \& Baumgart, T. Line Tension at Fluid Membrane Domain Boundaries Measured by Micropipette Aspiration. Physical Review Letters98, 208102, doi:10.1103/PhysRevLett.98.208102 (2007).

11 Portet, T., Gordon, S. E. \& Keller, S. L. Increasing membrane tension decreases miscibility temperatures; an experimental demonstration via micropipette aspiration. Biophys J103, L35-L37, doi:10.1016/j.bpj.2012.08.061 (2012).

12 Oglęcka, K., Rangamani, P., Liedberg, B., Kraut, R. S. \& Parikh, A. N. Oscillatory phase separation in giant lipid vesicles induced by transmembrane osmotic differentials. eLife3, e03695, doi:10.7554/eLife.03695 (2014).

13 Doeven, M. K. et al. Distribution, Lateral Mobility and Function of Membrane Proteins Incorporated into Giant Unilamellar Vesicles. Biophys J88, 1134-1142 (2005).

14 Hervás, J. H. et al. Human ATG3 binding to lipid bilayers: role of lipid geometry, and electric charge. Scientific Reports7, 15614, doi:10.1038/s41598-017-15057-6 (2017).

15 Hermann, E., Bleicken, S., Subburaj, Y. \& García-Sáez, A. J. Automated analysis of giant unilamellar vesicles using circular Hough transformation. Bioinformatics30, 1747-1754, doi:10.1093/bioinformatics/btu102 (2014).

16 Sych, T. et al. GUV-AP: multifunctional FIJI-based tool for quantitative image analysis of Giant Unilamellar Vesicles. Bioinformatics35, 2340-2342, doi:10.1093/bioinformatics/bty962 \% J Bioinformatics (2018). 
17 Zupanc, J. et al. Lipid Vesicle Shape Analysis from Populations Using Light Video Microscopy and Computer Vision. PLOS ONE9, e113405, doi:10.1371/journal.pone.0113405 (2014).

18 Usenik, P., Vrtovec, T., Pernuš, F. \& Likar, B. in 2010 20th International Conference on Pattern Recognition. 2520-2523.

19 Duda, R. O. \& Hart, P. E. Use of the Hough transformation to detect lines and curves in pictures. Commun. ACM15, 11-15, doi:10.1145/361237.361242 (1972).

20 Banani, S. F., Lee, H. O., Hyman, A. A. \& Rosen, M. K. Biomolecular condensates: organizers of cellular biochemistry. Nature Reviews Molecular Cell Biology18, 285-298, doi:10.1038/nrm.2017.7 (2017).

21 Banani, S. F. et al. Compositional Control of Phase-Separated Cellular Bodies. Cel/166, 651-663 (2016).

22 Brangwynne, C. P. et al. Germline P Granules Are Liquid Droplets That Localize by Controlled Dissolution/Condensation. Science324, 1729-1732, doi:10.1126/science.1172046 (2009).

23 Feric, M. et al. Coexisting Liquid Phases Underlie Nucleolar Subcompartments. Cel/165, 16861697 (2016).

24 Sanders, D. W. et al. Competing Protein-RNA Interaction Networks Control Multiphase Intracellular Organization. Cel/181, 306-324.e328, (2020).

25 Shin, Y. \& Brangwynne, C. P. Liquid phase condensation in cell physiology and disease. Science357, eaaf4382, doi:10.1126/science.aaf4382 (2017).

26 Nott, T. J. et al. Phase transition of a disordered nuage protein generates environmentally responsive membraneless organelles. Mol Cel/57, 936-947, doi:10.1016/j.molcel.2015.01.013 (2015).

27 Wegmann, S. et al. Tau protein liquid-liquid phase separation can initiate tau aggregation. The EMBO Journa/37, e98049, doi:10.15252/embj.201798049 (2018).

28 Yasuda, S. et al. Stress- and ubiquitylation-dependent phase separation of the proteasome. Nature578, 296-300, doi:10.1038/s41586-020-1982-9 (2020).

29 Huang, W. Y. C. et al. Phosphotyrosine-mediated LAT assembly on membranes drives kinetic bifurcation in recruitment dynamics of the Ras activator SOS. Proceedings of the National Academy of Sciences113, 8218-8223, doi:10.1073/pnas.1602602113 (2016).

30 Banjade, S. \& Rosen, M. K. Phase transitions of multivalent proteins can promote clustering of membrane receptors. eL ife3, e04123, doi:10.7554/eLife.04123 (2014).

31 Sharif Razavian, A., Azizpour, H., Sullivan, J. \& Carlsson, S. in Proceedings of the IEEE conference on computer vision and pattern recognition workshops. 806-813. 
32 Mitra, E. D., Whitehead, S. C., Holowka, D., Baird, B. \& Sethna, J. P. Computation of a Theoretical Membrane Phase Diagram and the Role of Phase in Lipid-Raft-Mediated Protein Organization. The Journal of Physical Chemistry B122, 3500-3513, doi:10.1021/acs.jpcb.7b10695 (2018).

33 Aghaaminiha, M., Ghanadian, S. A., Ahmadi, E. \& Farnoud, A. M. A machine learning approach to estimation of phase diagrams for three-component lipid mixtures. Biochimica et Biophysica Acta (BBA) Biomembranes1862, 183350 (2020).

34 Löpez, C. A., Vesselinov, V. V., Gnanakaran, S. \& Alexandrov, B. S. Unsupervised Machine Learning for Analysis of Phase Separation in Ternary Lipid Mixture. Journal of Chemical Theory and Computation15, 6343-6357, doi:10.1021/acs.jctc.9b00074 (2019).

35 Kraus, O. Z. et al. Automated analysis of high-content microscopy data with deep learning. 13, 924 (2017).

36 Guo, S.-M. et al. Revealing architectural order with quantitative label-free imaging and deep learning. eLife9, e55502, doi:10.7554/eLife.55502 (2020).

37 Hossain, M. S., Al-Hammadi, M. \& Muhammad, G. Automatic Fruit Classification Using Deep Learning for Industrial Applications. IEEE Transactions on Industrial Informatics15, 1027-1034, doi:10.1109/TII.2018.2875149 (2019).

38 Xuan, Q. et al. Multiview generative adversarial network and its application in pearl classification. ITIE66, 8244-8252 (2018).

39 Redmon, J. \& Angelova, A. in 2015 IEEE International Conference on Robotics and Automation (ICRA). 1316-1322 (IEEE).

40 Barros, P., Weber, C. \& Wermter, S. in 2015 IEEE-RAS 15th International Conference on Humanoid Robots (Humanoids). 582-587 (IEEE).

41 Zack, G. W., Rogers, W. E. \& Latt, S. A. Automatic measurement of sister chromatid exchange frequency. Journal of Histochemistry \& Cytochemistry25, 741-753, doi:10.1177/25.7.70454 (1977).

42 Tian, A. \& Baumgart, T. Sorting of lipids and proteins in membrane curvature gradients. Biophys J96, 2676-2688, doi:10.1016/j.bpj.2008.11.067 (2009).

$43 \mathrm{Au}$ - Olety, B., Au - Veatch, S. L. \& Au - Ono, A. Visualization of HIV-1 Gag Binding to Giant Unilamellar Vesicle (GUV) Membranes. JoVE, e54293, doi:doi:10.3791/54293 (2016).

\section{Figures}



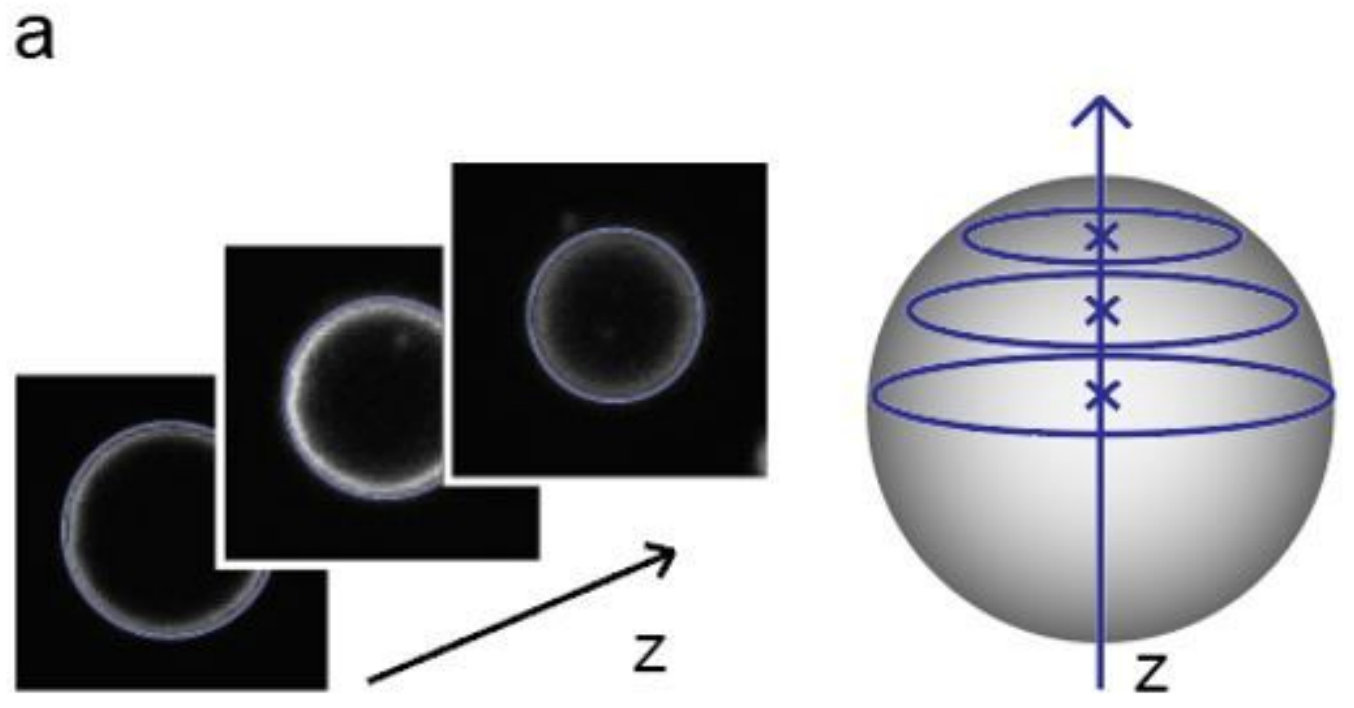

b Individual circular image filtering

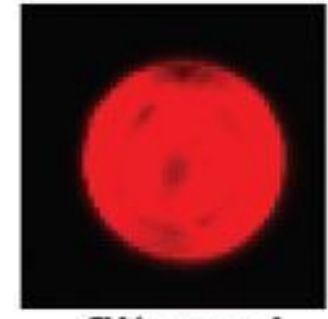

filtered

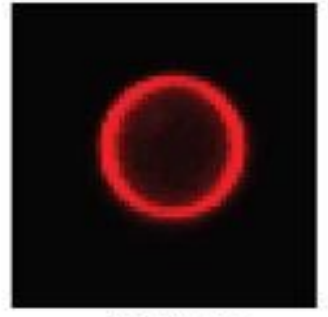

pass

\section{GUV stack filtering}

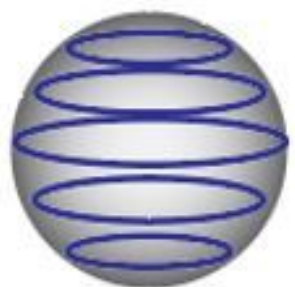

pass

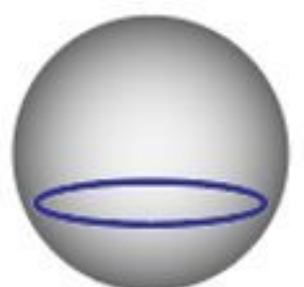

filtered

Figure 1

Schematic of GUV stack analysis. (a) Individual section images at different heights, or z positions are combined to construct a single whole GUV stack. The $(x, y)$ positions of the centers are used to determine whether the z-section images are from the same vesicle. (b) Filtering strategies can remove vesicles that do not strictly meet the quality to proceed with the final analysis. Individual circular image filtering makes decisions based on the quality of each circular section image. For example, a vesicle with too much multi- 
lamellar intensity can be removed. GUV stack filtering makes decisions based on the quality of a GUV stack as a unit, such as an insufficient number of images, to reconstruct a whole GUV.

a

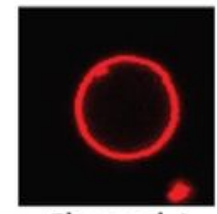

Channel A

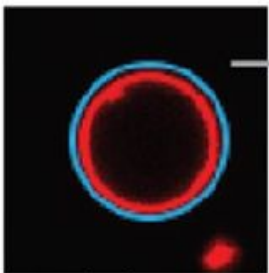

Circle detection and selection

b

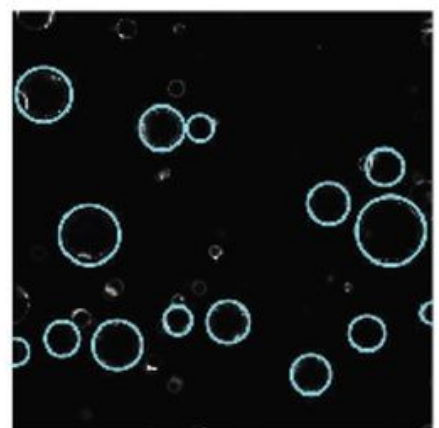

High SNR image detection

C

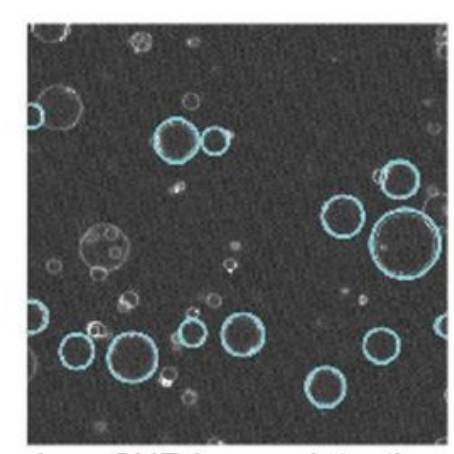

Low SNR image detection

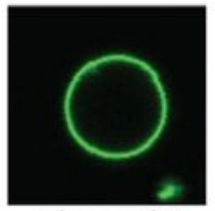

Channel B

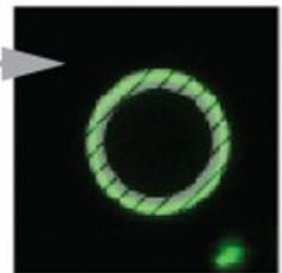

Sum up intensity near the circle

\section{Figure 2}

Circle detection and fluorescence intensity sampling. (a) The channel A fluorescence signal comes from the fluorescently tagged lipid molecules. The channel A signal is used to detect circles. Using the information on the position and radius of the detected fluorescence circles from channel $A$, the matching 
signal from channel $B$ is analyzed. The intensity signal of channel B comes from fluorescently tagged proteins that are supposed to bind to the lipid membrane. The net intensity of pixels that are close to the lipid membrane is summed up. (b) A typical example of a circle detection result when the signal-to-noise ratio of the fluorescence image is reasonably high. Blue circles indicate circles detected by the automated algorithm. (b) A typical example of a circle detection result when the signal-to-noise ratio of the fluorescence is relatively low. With the introduction of binary masking via noise thresholding, circles can still be successfully detected even from the low signal-to-noise ratio samples. The images shown in $b$ and c are $127.3 \mu \mathrm{m}$ in width and height. 


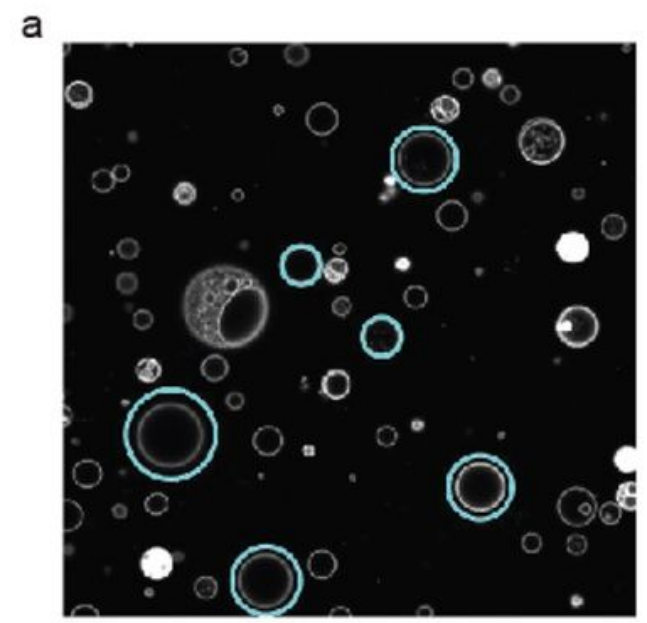

Uniform vesicles

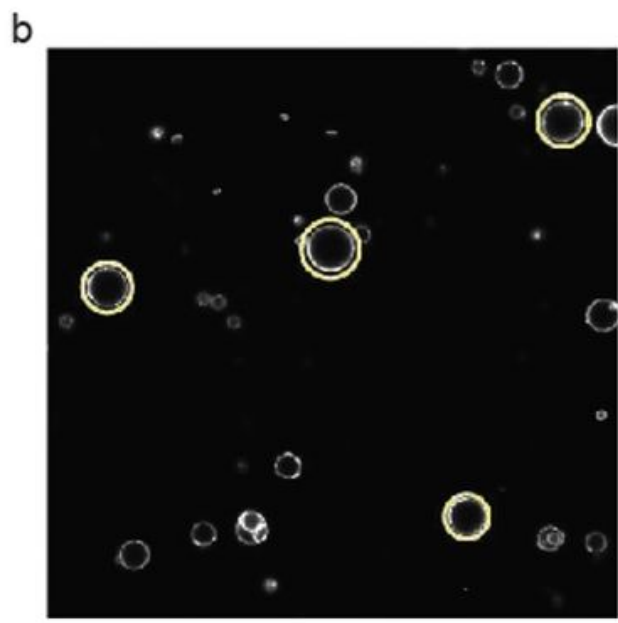

Phase separated vesicles

C
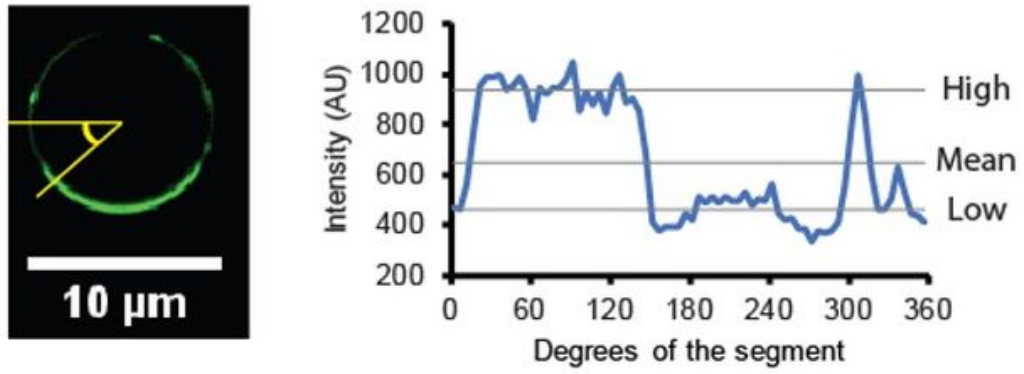

d
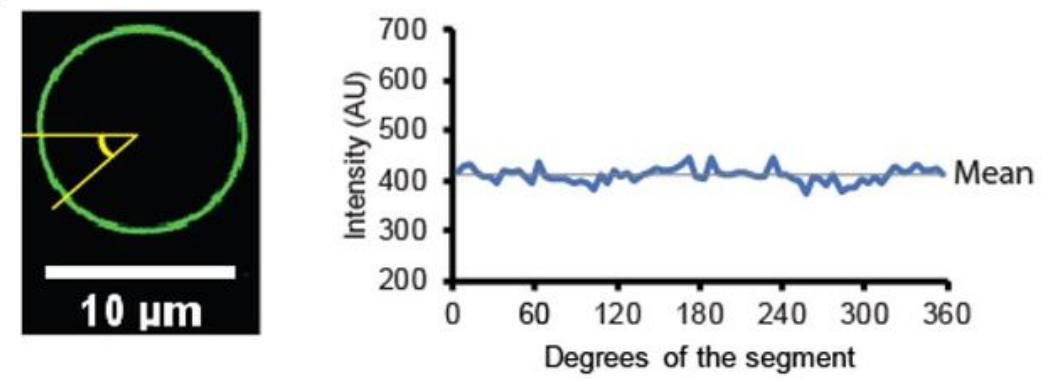

\section{Figure 3}

Contour intensity analysis for the determination of phase separation state. (a) A typical example of GUV detection from a uniform vesicle sample. Blue circles indicate the detected GUVs classified as uniform or no-phase domains. The composition of the vesicle was DOPC:Ni-DGS $=90: 10$ with $0.2 \mathrm{~mol} \%$ of TopFluorCholesterol replacing the DOPC. (b) A typical example of GUV detection from a phase-separated vesicle sample. Yellow circles indicate GUVs classified as phase separated or the coexistence of phase domains. 
The composition of the vesicle was DOPC:Ni-DGS:DPPC:Cholesterol $=15: 10: 50: 25$ with $0.2 \mathrm{~mol} \%$ of TopFluor-Cholesterol replacing the cholesterol. The images shown in a and b are $127.3 \mu \mathrm{m}$ in width and height. (c) An example intensity trace along the perimeter of a phase-separated vesicle. The intensity trace shows clear discontinuity at the boundaries of two-phase domains, which can be used to determine the existence of phase domains. The average intensity in each domain can also be estimated from the intensity analysis, as shown. (d) An example intensity trace along the perimeter of a uniform vesicle. The intensity trace shows no detectable discontinuity, which suggests the nonexistence of phase domains within the vesicle.

a

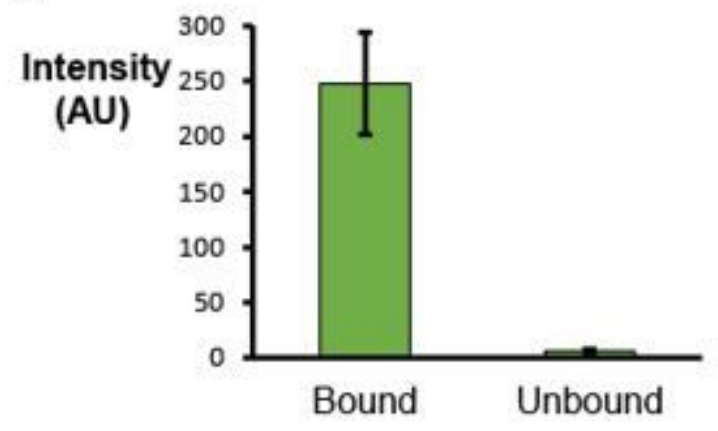

b

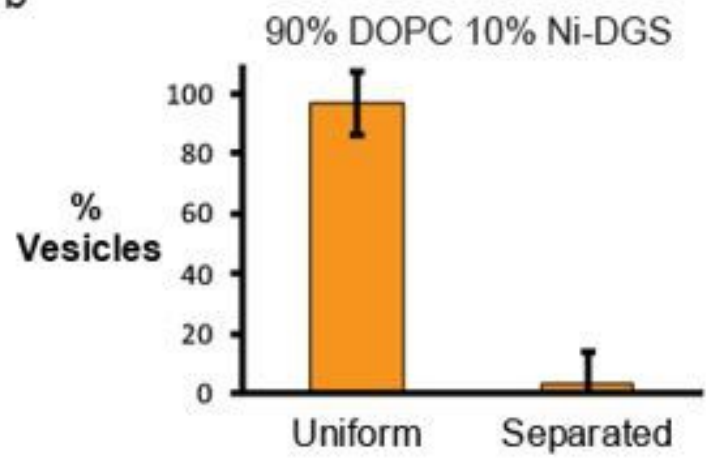

c

$15 \%$ DOPC $10 \%$ Ni-DGS

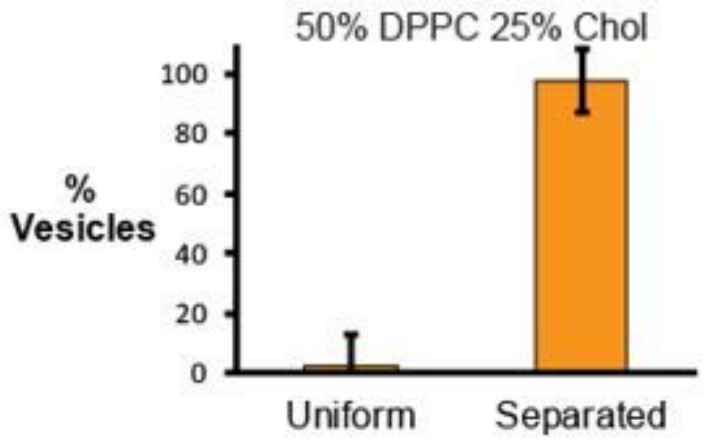

Figure 4 
Results of the example data analysis by the automated intensity trace calculation. (a) The fluorescence signals from the membrane-bound GFP proteins were quantified before and after the introduction of an inhibitor to detach the proteins from the membrane. The decrease in GFP intensity on the membrane is clear. (b) Uniform GUV samples with no phase domains were analyzed using the automated intensity contour-based phase state decision program. The \% of vesicles at each state, uniform and separated, was quantified. Most vesicles were in the uniform state. The $\%$ composition indicates the $\mathrm{mol} \%$ of the component lipids. (c) Phase-separated GUV samples with coexisting phase domains were analyzed using the automated intensity contour-based phase state decision. The \% of vesicles at each state, uniform and separated, was quantified. The \% composition indicates the mol \% of the component lipids. Most vesicles were in the phase-separated state with coexisting phase domains. Error bars indicate standard deviations of the quantity from image stacks. 

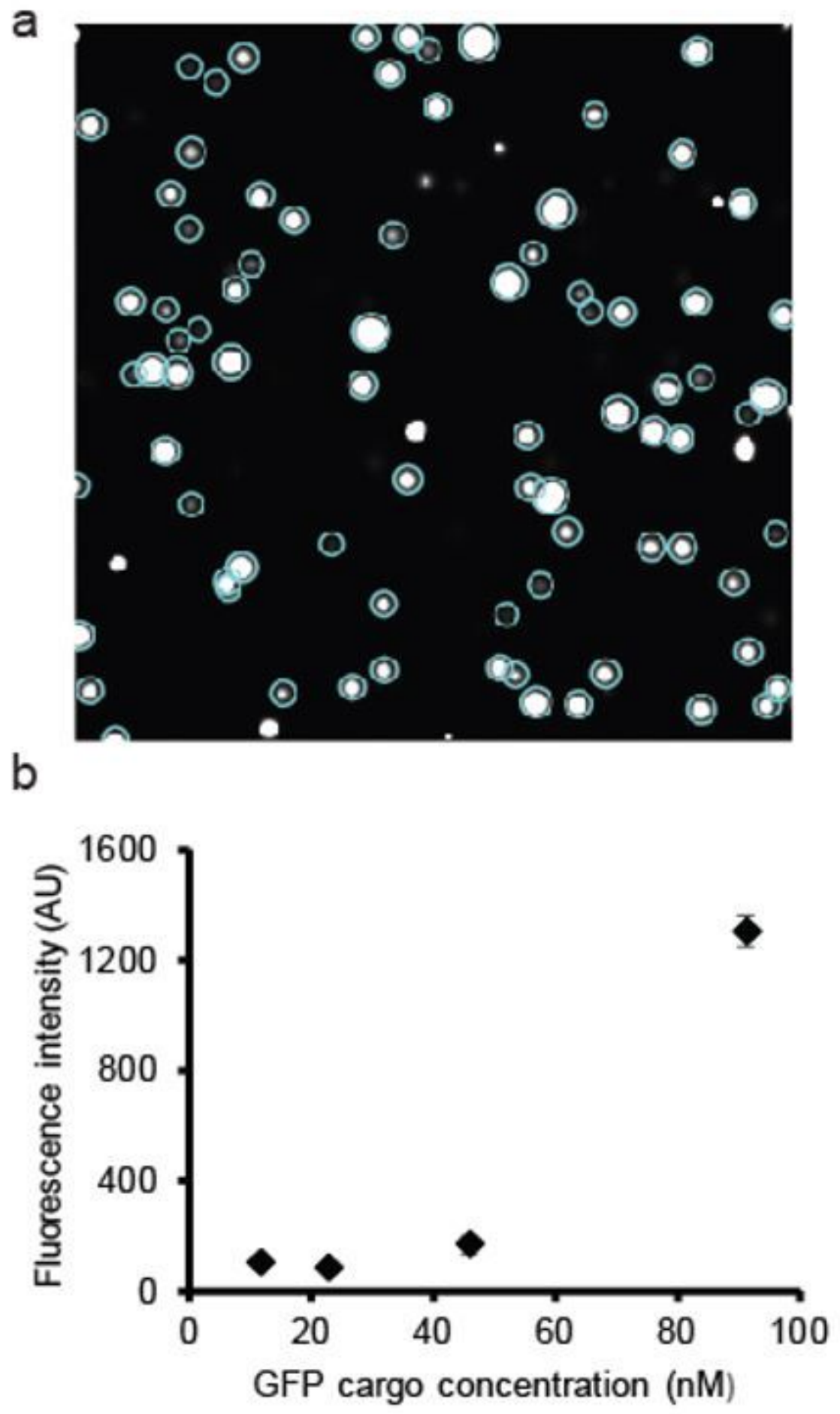

\section{Figure 5}

Automated protein liquid-liquid phase-separated droplet analysis. (a) CHT circle detection, followed by individual droplet grouping, efficiently detects well-behaved protein droplets from the sample. Blue circles indicate qualified protein droplets detected by the program. The image is $127.3 \mu \mathrm{m}$ in width and height. (b) The average fluorescence intensities per pixel within droplets were quantified for the same protein droplets at different fluorescent cargo protein concentrations. The fluorescence signal increases as the 
cargo concentration increases, although there was only a negligible deference between two data points below $25 \mathrm{nM}$. Error bars indicate standard deviations between image stacks.

a
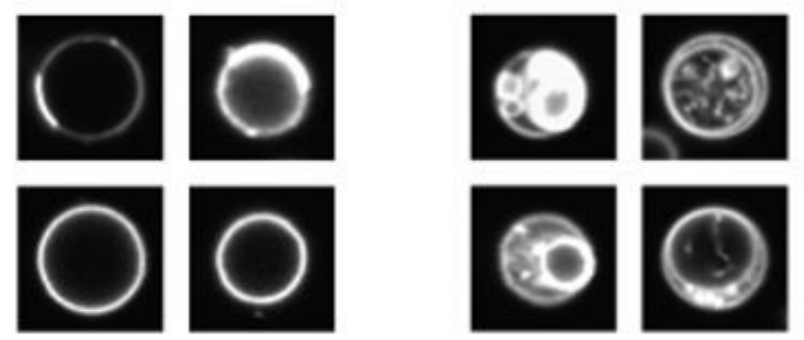

Class 1

Class 2

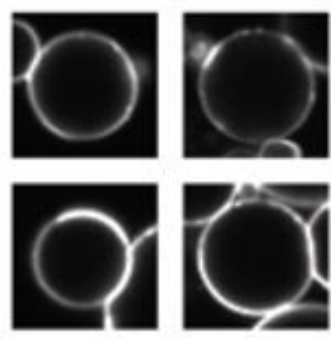

Class 3
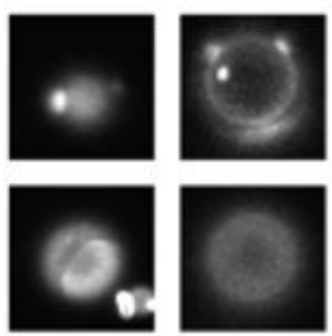

Class 4

b

C
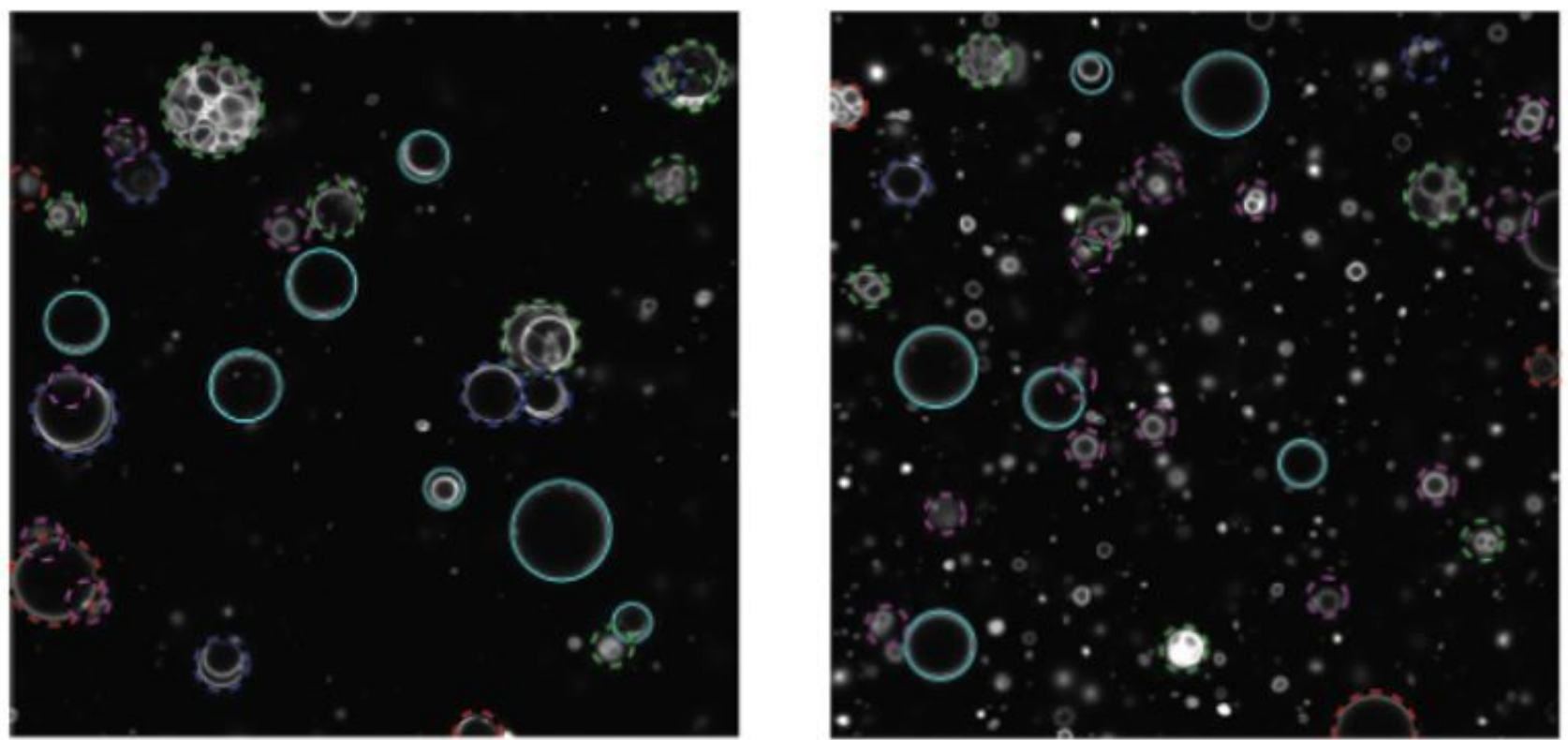

\section{Figure 6}

Deep learning-based vesicle selection filter. (a) Typical vesicle images used to train the neural network. Class 1 represents vesicles that are suitable for analysis. Class 2 represents multi-lamellar vesicles. Class 3 represents vesicles that are too closely overlapped with other vesicles, and class 4 represents vesicle images that are too hazy to analyze. (b, $c$ ) Example results of deep learning-based vesicle selection. Class 1 - cyan, Class 2 - green, Class 3 - blue, Class 4 - magenta, vesicles at the edges - red. Invalid vesicles were identified successfully for exclusion. The images are $127.3 \mu \mathrm{m}$ in width and height. 